\title{
Evaluación de la psicomotricidad en niños menores de 3 años durante la teleeducación en tiempos de confinamiento
}

\author{
Viviana del Rocío Arias Solis \\ https://orcid.org/0000-0002-8614-6056 \\ varias2877@uta.edu.ec \\ Lic. Mg. Esther del Rocío Benavides Rovalino \\ https://orcid.org/0000-0003-2188-2009 \\ edr.benavides@uta.edu.ec \\ Universidad Técnica de Ambato \\ Ambato - Ecuador
}

\section{RESUMEN}

Objetivo: Determinar la psicomotricidad en niños menores de 3 años, durante la teleducación en tiempos de confinamiento a través del test de Denver. La investigación fue de tipo básico con enfoque cuantitativo y de cohorte simple; se implementó el Test de Denver, se realizó de forma individual a cada niño. El test consta de 55 ítems, y clasifica a los niños dependiendo de sus fallas como normal, dudoso y anormal. Se incluyeron 100 participantes a la población de estudio como resultado los niños de 0 a 3 años en el área personal social el 95\% tiene un desarrollo normal y el 5\% presenta retraso; en el área de motricidad fina el 51\% alcanza el desarrollo adecuado y el $49 \%$ presenta un retraso; dentro del área motricidad gruesa el 34\% presenta retraso y el 66\% logra un desarrollo adecuado; en el área de lenguaje el 58\% tiene un desarrollo normal y el $42 \%$ presenta retraso. En conclusión, la pandemia originada por el Covid-19 está asociada con el incremento de factores de riesgo para el adecuado desarrollo de la psicomotricidad. Se ha logrado una mediación con la tecnología y las posibilidades de concretar un enfoque inclusivo y personalizado en la educación virtual.

Palabras clave: Aislamiento Social; educación; niño; personal de salud; Ecuador. 


\title{
Psychomotricity evaluation in children under 3 years of age during the tele-education in times of confinement
}

\begin{abstract}
Objective: To determine the psychomotor skills in children under 3 years of age, during tele-education in times of confinement through the Denver test. The research was of a basic type with a quantitative approach and a simple cohort; The Denver Test was implemented, and it was also carried out individually for each child. The test consists of 55 items, and classifies children depending on their failures: normal, doubtful and abnormal. One hundred participants were included in the study population. As a result, children from 0 to 3 years old in the personal social area, 95\% had normal development and $5 \%$ presented delay; in the area of fine motor skills, $51 \%$ reach adequate development and $49 \%$ present a delay; within the gross motor area, $34 \%$ present retardation and $66 \%$ achieve adequate development; In the language area, 58\% have a normal development and $42 \%$ present delay. In conclusion, the pandemic caused by Covid-19 is associated with an increase in risk factors for the adequate development of psychomotor skills. Mediation has been achieved with technology and the possibilities of realizing an inclusive and personalized approach in virtual education.
\end{abstract}

Keywords: Social Isolation; education; child; health personnel; Ecuador

Artículo recibido: 15 octubre. 2021 Aceptado para publicación: 18 noviembre 2021

Correspondencia: Varias2877@uta.edu.ec Conflictos de Interés: Ninguna que declarar 


\section{INTRODUCCIÓN}

El concepto psicomotricidad supone abordar la significación del cuerpo a lo largo de la historia; desde las primeras civilizaciones hasta nuestros días, ya que el cuerpo ha sufrido innumerables transformaciones a través del tiempo, hasta que Descartes influenciado por su pensamiento científico llega a considerar al cuerpo como una "pieza del espacio visible", separado del "sujeto pensante".

Actualmente la psicomotricidad es una disciplina que, basándose en una concepción integral del ser humano se ocupa de la interacción que se establece entre el conocimiento, la emoción, el cuerpo y el movimiento, además de su importancia para el desarrollo de la persona (Mendiara, 2016).

La evolución psicomotriz de niñas y niños depende de la maduración neurológica, pues el desarrollo de los órganos es paralelo al motor, y es de evolución rápida. Partiendo de los movimientos básicos (desplazamiento, manipular objetos, postura, etc.), para llegar a conductas motrices (marcha, carrera, saltos, lanzamiento, etc.) mismos que desencadenan la facilidad de movimientos más complejos de algunos juegos y deportes.

Una percepción temprana del mundo que nos rodea nos permite construir esquemas mentales en un entorno más inmediato, esta exploración es posible gracias al desarrollo del movimiento, a su vez esta permite la adquisición de capacidades que darán como resultado al desarrollo cognitivo (Carbonero, 2016). El desarrollo psicomotriz es considerado como una adquisición progresiva de habilidades que genera cada persona, y existen etapas cruciales como lo es la niñez, en donde puede existir un riesgo en la aparición de problemas de este desarrollo (Camargos, 2016).

El 11 de marzo del 2020 la Organización Mundial de la Salud (OMS), declara una emergencia epidemiológica a nivel mundial debido a la presencia del SARS-CoV-2. A causa de la presencia de este virus mortal el Ecuador y el mundo entero se declara estado de emergencia tomando medidas preventivas como el aislamiento social, restricciones de diferentes centros educativos, centros de diversión, comerciales, parques; y a pesar de que los diferentes establecimientos de salud permanecían en funcionamiento, debido a este confinamiento se limitó a los niños, adultos y ancianos a permanecer en sus hogares para salvaguardar sus vidas; por lo que diferentes establecimientos educativos optaron por impartir el conocimiento a través de clases virtuales; no obstante ya se apreciaba el déficit en el desarrollo de los niños que permanecían en el subregistro, captados por el personal 
de salud por lo que asistían a estimulación y consecuentemente por las medidas de confinamiento podría haber afectado el correcto desarrollo de los niños durante su primera infancia (Sánchez, Ramón \& Mayorga, 2020).

\section{ESTRATEGIAS METODOLÓGICAS O MATERIALES Y MÉTODOS}

La metodología de la investigación es de tipo básico debido a que se origina en un marco teórico, con la finalidad de incrementar los conocimientos científicos y ampliar la información de otras investigaciones de la misma naturaleza, con enfoque cuantitativo que permitirá recolectar y analizar datos numéricos generados a través de la aplicación de un test; y de cohorte simple (Escobar et al., 2018); ya que se pretende analizar la psicomotricidad de los niños menores de 3 años que asisten a la estimulación en el centro de salud Totoras, lo cual permitirá identificar afecciones del desarrollo psicomotriz generados por el confinamiento.

La población utilizada en el estudio son niños que asisten al programa de estimulación en el Centro de Salud Totoras, perteneciente a la ciudad de Ambato en Ecuador, mismo que cuenta con 100 niños menores de 3 años.

En este estudio se implementó el Test de Denver que será aplicada a toda la población. El test de Denver es una prueba evolutiva que tiene como objetivo principal medir las habilidades físicas, intelectuales y de desarrollo psicomotriz de los niños, este instrumento fue creado por tres profesionales de psicología, Williams Frankenburg, Josieh B. Doods y Alma Fordal, en el años 1967 en el Centro Médico de la Universidad de Colorado Denver (Aguilar, 2017), está diseñado en base de cuatro áreas específicas: área personal social, área de motricidad fina, área del lenguaje, área de motricidad gruesa que se aplica a niños de 0 a 6 años; el tiempo de administración de la prueba fue de aproximadamente 20 minutos, se realizó de forma individual a cada niño que acude a la estimulación en el centro de Salud Totoras (Rivera \& Salto, 2017). Para ejecutar la prueba fue importante crear un ambiente agradable, en donde estuvo presente la madre o familiar del niño. El test consta de 55 ítems, pero sólo se debe aplicar aquellos que se encuentran a la izquierda de la línea de edad, hay que preguntar a la familia si el niño realiza los elementos que quedan a la izquierda de la línea trazada o aquellos que atraviesan la línea. En caso de que el niño o niña falla en las actividades de la prueba que normalmente es realizada por el $90 \%$ de los niños de su edad cronológica, debe considerarse como un fallo absoluto; y se considera fallo relativo cuando el niño no supera una prueba que hace el 
$75 \%$ de los niños/as de su edad. En la valoración del test sólo se contabilizan los fallos absolutos, y estos pueden valorarse como problemático o dudoso. Se considera problemático cuando haya dos o más fallos, en dos o más áreas; y dudoso si sólo un área tiene 2 o más fallos (Boo, Mateus \& Durya, 2018). "La fiabilidad del test es del 90\% y entre evaluadores del $80-95 \%$ es de fácil aplicación y no es costoso, su sensibilidad es de 56 al 83\% y una especificidad entre el 43 al 80\%" (Cruz, Vega \& Gonzales, 2019).

El procesamiento de la información se realizó a través de una tabulación en graficas de barras utilizando Excel, el cual es una hoja de cálculo que nos permite manipular datos numéricos y de texto, para analizar información (Grajales, 2016); se tabuló los resultados obtenidos en el Test de Denver, mismo que clasificó a los niños dependiendo de sus fallas como normal, dudoso y anormal.

\section{RESULTADOS Y DISCUSIÓN}

Entre el 1 al 31 de octubre del 2021 se incluyeron 100 participantes a la población de estudio, misma que está conformada por 15 niños de 0 a 1 año, 54 niños de 1 a 2 años y 31 niños de 2 a 3 años que asisten a la estimulación en el centro de salud Totoras.

En la (figura 1) se puede observar que dentro del área personal social los niños entre 0 y 1 año obtienen un Denver normal de acuerdo a su edad, de los niños de 1 a 2 años, 50 niños consiguen un Denver adecuado y 4 no realizan las actividades dentro del test por lo que son considerados dudosos, de 2 a 3 años, 30 niños presentan un Denver normal y 1 niño presenta retraso por lo que es considerado dudoso.

Figura 1. Evaluación del área Personal social, Centro de Salud Totoras, octubre 2021

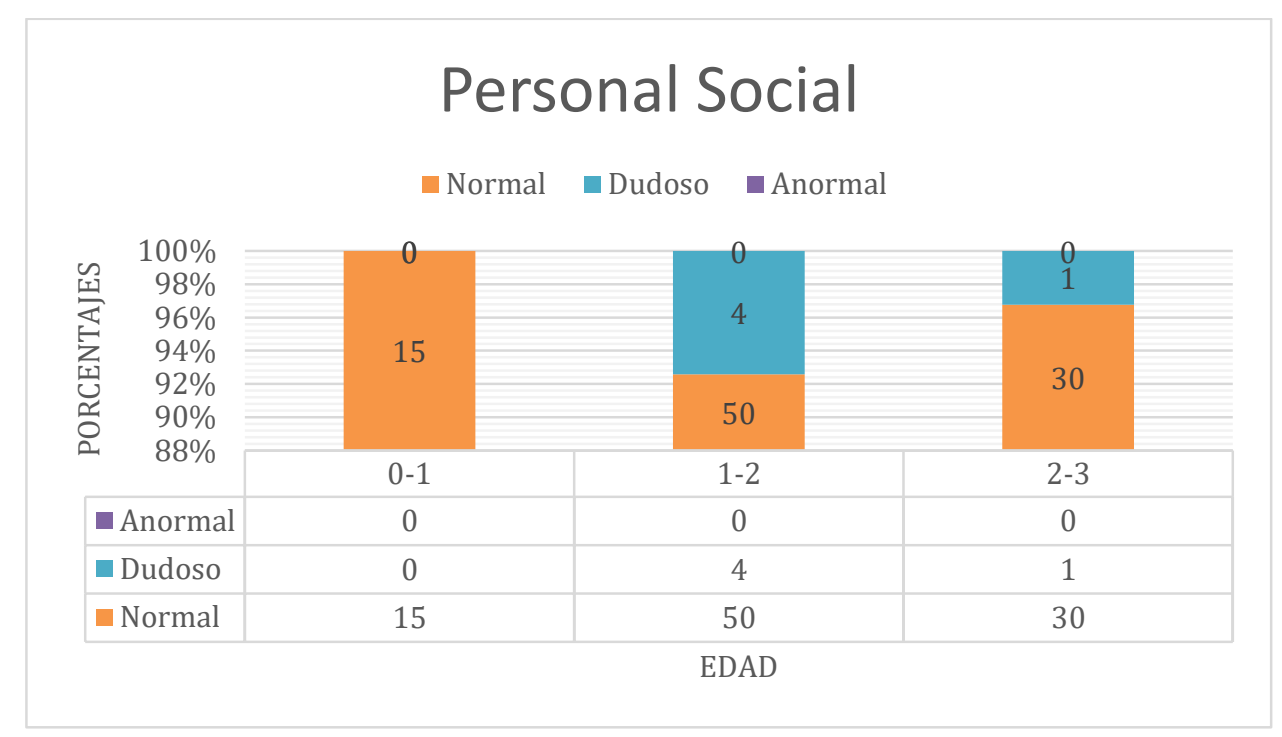

Fuente: Elaboración propia de la autora a partir de los resultados obtenidos 
En la (figura 2) correspondiente al área motricidad fina se aprecia un retraso dentro de los 3 rangos de edades, de 0 a 1 año, 9 niños se encuentran dentro de un Denver normal, mientras que 6 niños están dentro de un Denver dudoso, de 1 a 2 años, 31 niños presentan un Denver adecuado y 23 niños presentan retraso por lo que son dudosos, de 2 a 3 años los niños con retraso son 20 resultando ser más elevado que los niños con Denver normal que dentro de este rango solo fueron 11.

Figura 2. Evaluación del área Motricidad fina, Centro de Salud Totoras, octubre 2021

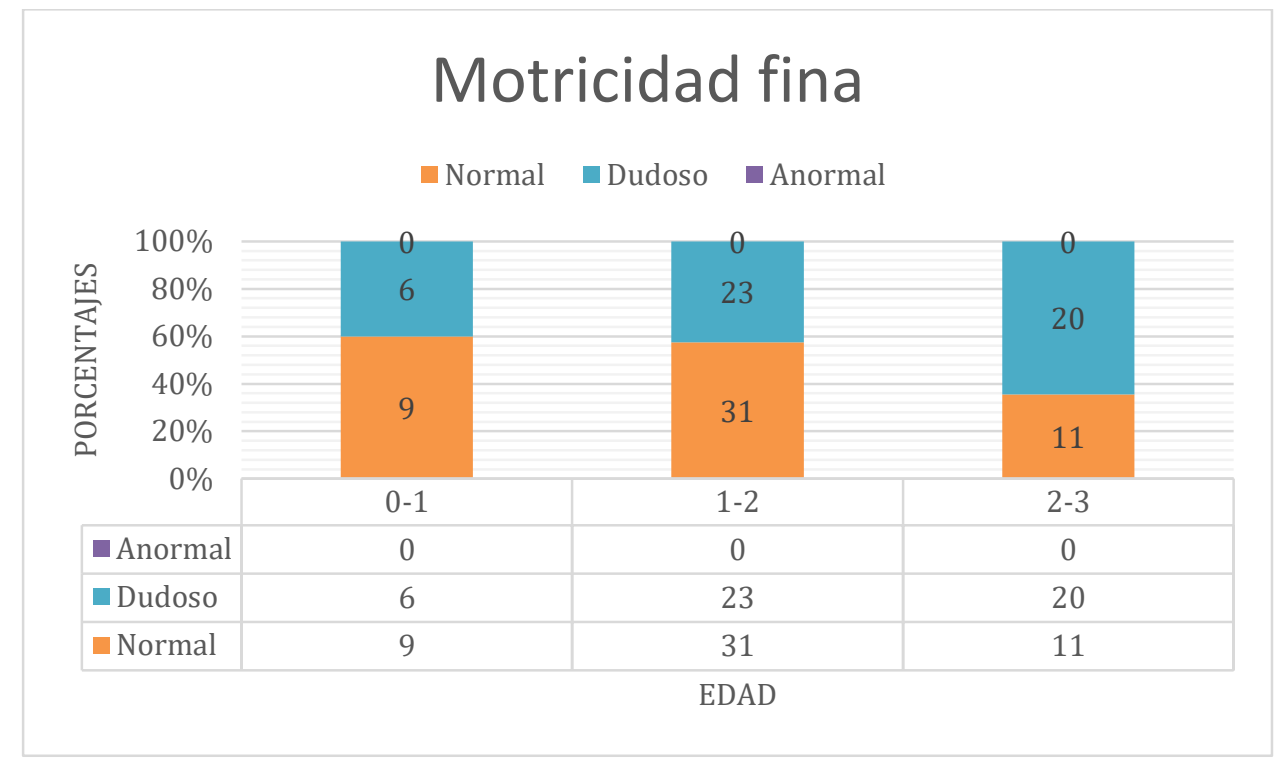

Fuente: Elaboración propia de la autora a partir de los resultados obtenidos

En el área del lenguaje que se aprecia en la (figura 3) los niños de 0 a 1 año se encontraban con un desarrollo normal, dentro de los niños de 1 a 2 años, 14 obtuvieron un Denver normal, mientras que 40 niños presentaron un retraso evidente, por otro lado, los niños de 2 a 3 años, 18 niños de 31 obtuvieron un Denver dudoso. Finalmente, dentro del área de motricidad gruesa (figura 4) podemos evidenciar que, dentro del rango de 0 a 1 año de edad, 5 niños de 15 presentaron un retraso mientras que, de 1 a 2 años 13 niños de 41 obtuvieron un Denver dudoso y de los niños de 2 a 3 años, 16 presentaron retraso y 15 un Denver normal. 
Figura 3. Evaluación del área Lenguaje, Centro de Salud Totoras, octubre 2021

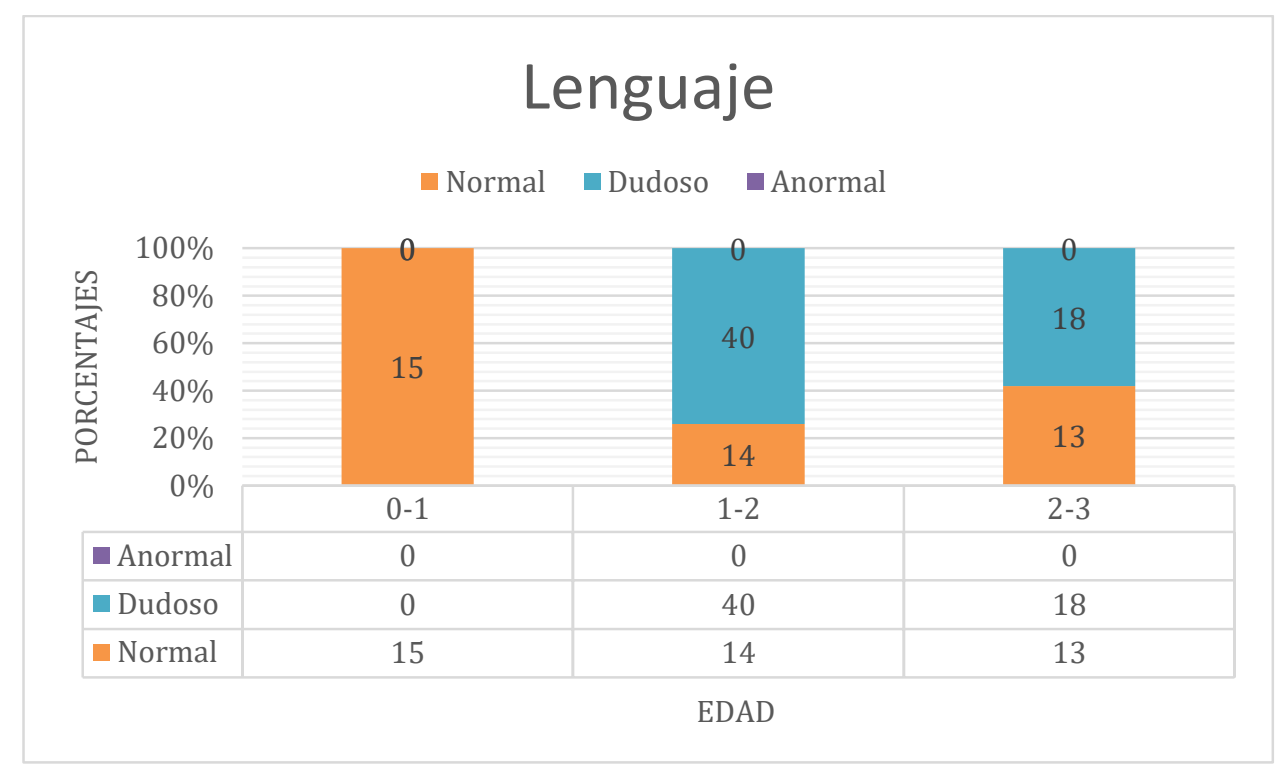

Fuente: Elaboración propia de la autora a partir de los resultados obtenidos

Figura 4. Evaluación del área Motricidad gruesa, Centro de Salud Totoras, octubre 2021

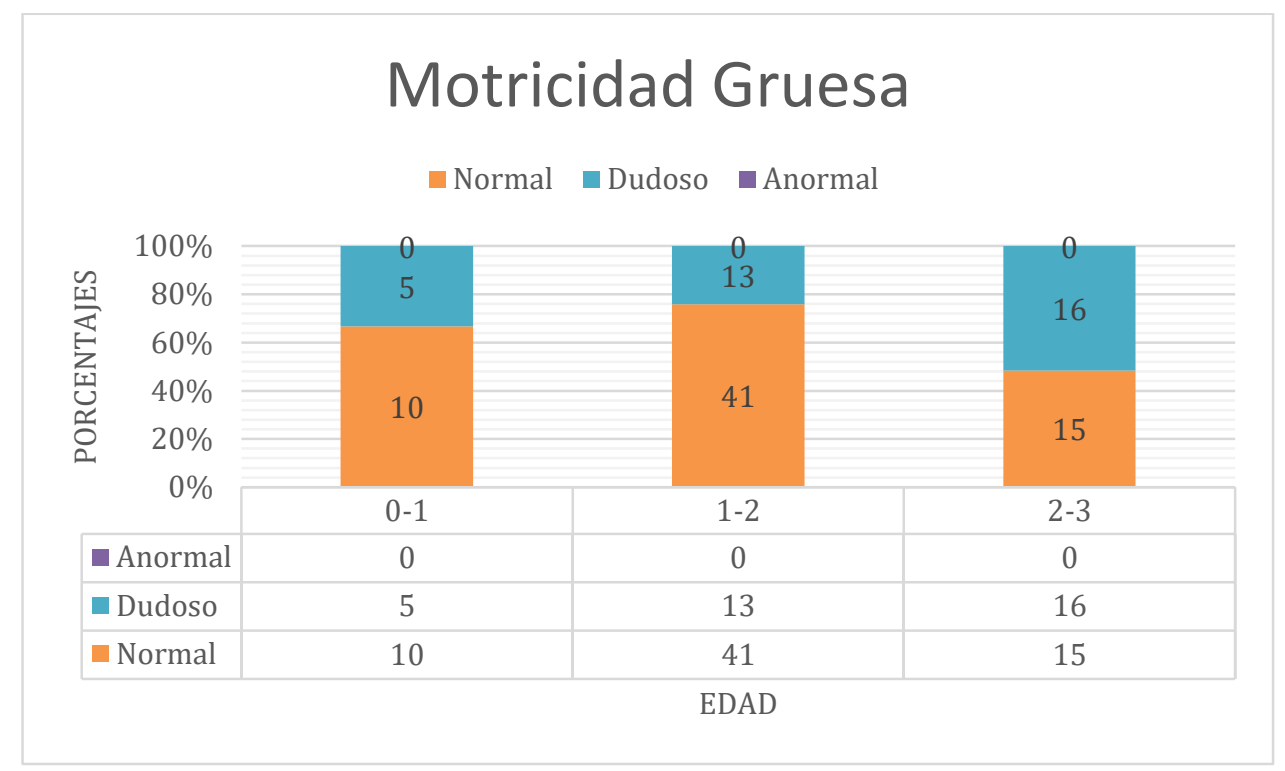

Fuente: Elaboración propia de la autora a partir de los resultados obtenidos

De forma general se puede evidenciar en la (figura 5), que los niños de 0 a 3 años en el área personal social el 95\% tiene un desarrollo normal y el 5\% presenta retraso; en el área de motricidad fina el 51\% alcanza el desarrollo adecuado y el $49 \%$ presenta un retraso; dentro del área motricidad gruesa el 34\% presenta retraso y el 66\% logra un desarrollo adecuado; en el área de lenguaje el 58\% tiene un desarrollo normal y el $42 \%$ presenta retraso. 
Figura 5. Resultados generales de la evaluación del Test de Denver de 0 a 3 años de edad, Centro de salud Totoras, octubre 2021

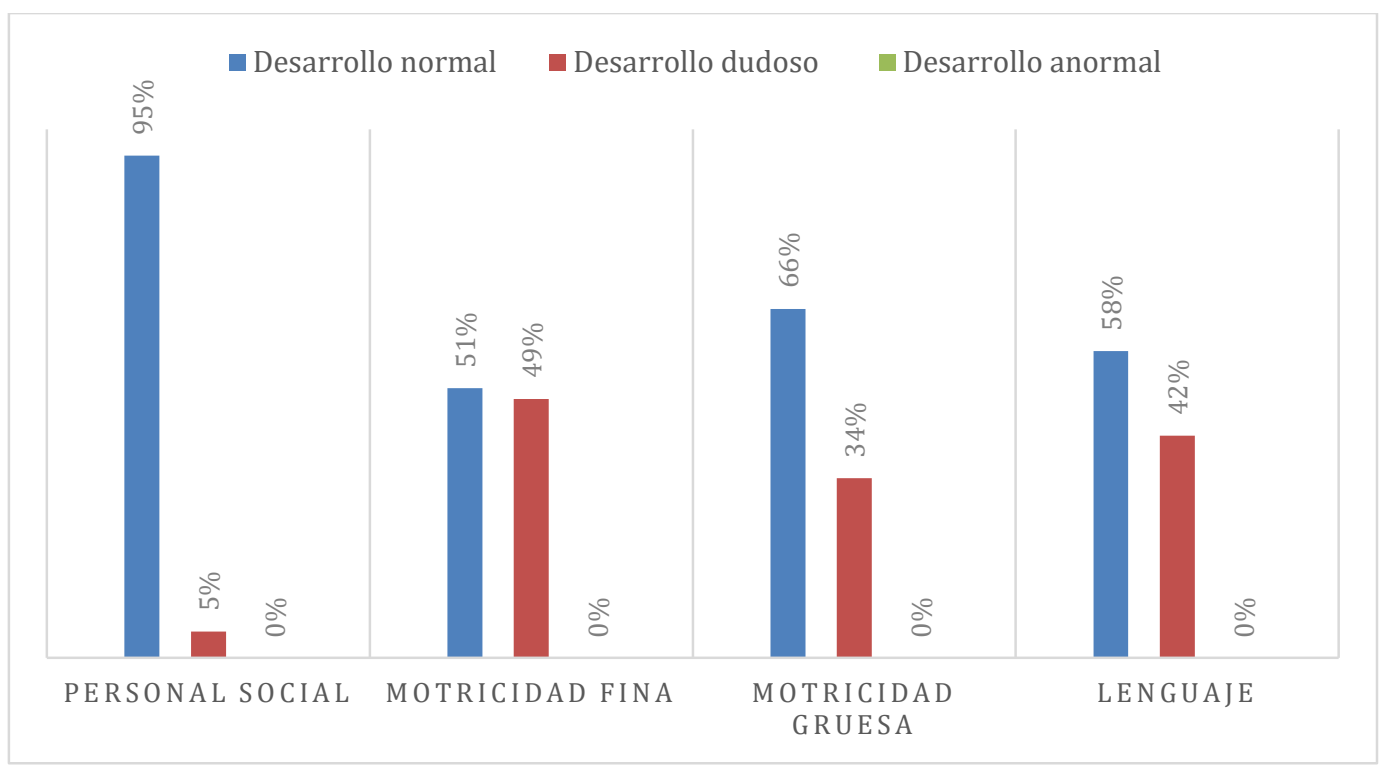

Fuente: Elaboración propia de la autora a partir de los resultados obtenidos

\section{DISCUSIÓN}

En la evaluación aplicada mediante el Test de Denver refleja que existe un mayor retraso en la motricidad fina con un $49 \%$ de la población total, esta área comienza a desarrollarse desde la vida intrauterina, y su desarrollo global depende de la relación entre las características heredadas y experiencias vividas; los cambios constantes son una fuente que influyen en el mismo. La motricidad fina implica precisión, eficacia, y armonía; esto incluye realizar acciones como alcanzar, agarrar y manipular objetos desarrollando destrezas con las manos y dedos de manera precisa de acuerdo con la exigencia de la actividad (Serrano \& Luque, 2019). A diferencia del desarrollo de la motricidad gruesa que se aprecia en el esquema del cuerpo mediante los músculos grandes, pero siempre va en constante relación con el desarrollo de la motricidad fina.

Muchos psicopedagogos creen en teorías de aprendizaje que relacionan el esquema corporal y la motricidad gruesa a manera de punto de inicio para un buen manejo de procesos senso-perceptivos en el aprendizaje, que se va dando de forma céfalo-caudal empezando por el cuello, posteriormente el tronco la cadera y finalmente en las piernas, dichas fases se ven reflejadas en el control de la cabeza, aprender a sentarse, gatear, caminar, coordinar, ritmo y mantener el equilibrio (Díaz \& Sysley, 2019); en nuestra población de estudio los niños con más retraso fueron los que estaban dentro de los 2 a 3 
años de edad, ya que 16 niños de 31 no desarrollaban la capacidad de coordinar, mantener el equilibrio, caminar correr y saltar.

Esta área de la psicomotricidad al igual que las otras áreas son influenciadas por el ambiente que se proporcione al niño, sin embargo, según los resultados obtenidos en la evaluación, el retraso en el desarrollo de la motricidad fina ha afectado en mayor proporción a los niños de la población estudiada. Varios autores indican que la motricidad se ve condicionada por la disminución de actividad física y la limitación de áreas utilizadas (Poblete, Guerra, Toro \& Cruzat, 2016), este factor de riesgo tuvo un mayor impacto durante el confinamiento, por lo que la motricidad de los niños se ha visto afectada al limitarles diferentes áreas recreativas y centros pedagógicos a los que asistían antes del confinamiento.

Otra área con un significante retraso que ha aumentado su incidencia fue el área de lenguaje con un $42 \%$ de retraso en la población total, estos niños de acuerdo a su rango de edad no podían expresar sus deseos con palabras y no pronunciaban más de 6 palabras y a su vez no han podido recibir una buena estimulación de lenguaje por la pandemia.

El nivel de desarrollo psicomotor del niño avanza rápidamente y de ahí la importancia de proporcionar al niño un ambiente adecuado en estimulación sensorial y perceptiva mediante pautas de activación de lo contrario se evidencia un retraso (Flores, 2013).

El área con menos incidencia de retraso en la población es la de personal-social, representando un mínimo retraso del $5 \%$, podría deberse a la interacción prolongada con los miembros de la familia durante el confinamiento, por lo que se considera que posiblemente haya una correlación positiva al interactuar el niño eficazmente en un grupo pequeño, nutriendo las situaciones de identidad y la capacidad de relacionarse con los que lo rodean (Rubio, García \& Cervantes, 2011).

La pandemia por el COVID-19 ha evidenciado una fragilidad y vulnerabilidad en los hogares tanto a nivel material como emocional, mediante el estudio presente podemos considerar que ha afectado fuertemente la calidad de vida y las condiciones de educación, estimulación de niñas/niños y padres de familia (Ponce, Bellei \& Vielma, 2020).

El confinamiento según varios autores podría conllevar a graves problemas en todas las áreas del desarrollo. Uno de los pocos estudios enfocados en la evaluación del desarrollo psicomotriz en el contexto de la pandemia se encuentra realizado por Ceballos (Ceballos $\&$ Sevilla, 2020). El cual es un estudio cualitativo y descriptivo realizado en niños de 
Argentina en donde se evidenció que a pesar de los esfuerzos en la enseñanza por parte de educadores existió mayor prevalencia de los déficits en las áreas del desarrollo (Sánchez et al., 2020), generando así una relación directa con la dificultad de enseñanza para una correcta estimulación, lo cual conlleva a cuestionar la eficacia de la teleeducación.

Actualmente y debido a la emergencia sanitaria generada por el Covid-19 el uso de la tecnología ha sido una opción que ha obligado a los docentes a replantear sus prácticas pedagógicas llevando a interesantes escenarios que transforman sin lugar a duda su práctica, utilizando las TIC (Tecnologías de la Información y Comunica) (Ruiz, Sacán \& Vintimilla, 2021).

A pesar de que se considera que los docentes son capaces de hacer uso de tecnologías e información para el desarrollo de herramientas virtuales de aprendizaje tomando en consideración aspectos éticos y morales (Martínez \& Garcés, 2020); son los padres quienes no alcanzan el nivel necesario para adecuarse a un sistema de educación virtual, influyendo factores económicos como la falta de recursos tecnológicos, según un estudio de experiencias educativas en casa durante la pandemia COVID- 19 realizado en Chile, señala que aproximadamente un $90 \%$ de los hogares cuentan con dispositivos con capacidad para conectarse a internet ya sea un celular, computador o tablet, pero hay una importante variación en la calidad de dicha conexión, indicando que aproximadamente 3 de cada 5 hogares tienen conexión fija, 1 de cada 3 tiene algún tipo de conexión móvil, por lo que sólo unos de cada tres participantes tienen un acceso optimo y un cuarto de los hogares tendrían una conexión deficiente, ya sea porque es poco estable y/o demasiado lenta; además de la poca adaptabilidad al pasar de la práctica y enseñanza tradicional, llevándolo a manera virtual hace que las practicas pedagógicas sean menos atractivas e interactivas; conllevando a una escasa participación y falta de interés por parte de los niños y padres de familia (Ruiz et al., 2021).

El uso de la tecnología por sí sola no genera innovación, todos los dispositivos desarrollan procesos y establecen relaciones distintas con los usuarios, según el vínculo y la forma en la que transmite el mensaje (Briceño, 2019), por lo que se requiere del rol del docente para generar ambientes y experiencias de aprendizaje virtual al igual que se requiere del compromiso de los padres ayudar a sus niños en los talleres pedagógicos para el desarrollo adecuado de la psicomotricidad durante la emergencia sanitaria presente, de otro modo 
se verá reflejado en el retraso de las diferentes áreas de la psicomotricidad como se ha comprobado en el presente estudio.

\section{CONCLUSIÓN O CONSIDERACIONES FINALES}

La pandemia originada por el Covid-19 esta generalmente asociada con el incremento de los factores de riesgo para el adecuado desarrollo de la psicomotricidad en los niños y niñas; ya que experimentar una emergencia sanitaria y un aislamiento social en estos primeros años de vida, marcan notablemente el desarrollo motor, físico mental y social debido a que los niños son muy sensibles a todos los estímulos de su alrededor; y a pesar de los aciertos y desafíos que impone la educación virtual se ha logrado una mediación con la tecnología y las posibilidades de concretar un enfoque inclusivo y personalizado en la práctica pedagógica de la estimulación psicomotriz, no obstante, se siguen evidenciando dificultades con esta modalidad.

Agradecimientos: Agradezco al Director del Distrito 18D02 ZONAL3-SALUD perteneciente al Ministerio de Salud Pública por permitir la realización de esta investigación en el centro de Salud Totoras Ambato.

\section{LISTA DE REFERENCIAS}

Aguilar, D. L. A. (2017). Paola Andrea Narváez Molina. 58.

Boo, F. L., Mateus, M. C., \& Duryea, S. (2018). Analysis of socioeconomic gradients in the development of children aged 0-3 years in Fortaleza, Northeastern Brazil. Revista de Saúde Pública, 52, 84-84. https://doi.org/10.11606/s15188787.2018052000525

Briceño, L. (2019). Usos de las tic's en preescolar: Hacia la integración curricular. Panorama, 13(24), 20-32. Recuperado de https://dialnet.unirioja.es/servlet/articulo?codigo $=6888662$

Camargos, E. K. (2016). La importancia de la psicomotricidad en la educación de los niños. Revista Científica Multidisciplinar Núcleo do Conhecimento. https://www.nucleodoconhecimento.com.br/educacion-es/psicomotor-en-laeducacion-y-el-nino

Carbonero Celis, C. (2016). Sensomotricidad y psicomotricidad en la infancia. Sevilla Wanceulen Editorial, p.

Ceballos M. N., \& Sevilla V. S. (2020). El efecto del aislamiento social por el Covid-19 en la conciencia emocional y en la comprensión lectora. 9, 1-13. 
Cruz, G. C. P., Vega, E. S. M., \& Gonzales, R. A. C. (2019). Aplicación del test de Denver II en la evaluación del desarrollo infantil. RECIMUNDO: Revista Científica de la Investigación y el Conocimiento, 3(Extra 3 (ESP)), 25-37.

Diaz R. J., Sisley T. L., (2019). Motricidad Gruesa. Recuperado de http://repositorio.ucp.edu.pe/bitstream/handle/UCP/910/SISLEY_DIAZ_EDU_T RABINV_BACH_2019.pdf?sequence=1\&isAllowed=y

Escobar, A. A. H., Rodríguez, M. P. R., López, B. M. P., Ganchozo, B. I., Gómez, A. J. Q., \& Ponce, L. A. M. (2018). METODOLOGÍA DE LA INVESTIGACIÓN CIENTÍFICA. 3Ciencias.

Flores, J. (2013). Efectividad del programa de estimulación temprana en el desarrollo psicomotor de niños de 0 a 3 años. Revista CIENCIA Y TECNOLOGÍA, 9(4), 101-117.

Grajales, L. (s. f.). Manual de Word, Excel y Power Point Avanzado. 97. Recuperado de http://web.seducoahuila.gob.mx/biblioweb/upload/Manual\%20de\%20Word\%20 y\%20Exce1\%20avanzados.pdf

Martínez-Garcés, J., \& Garcés-Fuenmayor, J. (2020). Competencias digitales docentes y el reto de la educación virtual derivado de la covid-19. Educación y Humanismo, 22(39), 1-16. https://doi.org/10.17081/eduhum.22.39.4114

Mendiara Rivas, J. (2016). Psicomotricidad educativa. Sevilla: Wanceulen Editorial

Poblete, F., Guerra, R., Toro, P., \& Cruzat, E. (2016). DESARROLLO MOTOR GRUESO EN ESCOLARES DE ZONA URBANA Y RURAL. Revista Horizonte Ciencias de la Actividad Física, 7(1), 59-66.

Ponce, T. Bellei, C. Vielma, C. (2020). Experiencias educativas en casa de ninas y ninos durante la pandemia COVID19.pdf. (s.f.). Recuperado de https://bibliotecadigital.mineduc.cl/bitstream/handle/20.500.12365/17285/Experi encias $\% 20$ educativas $\% 20 \mathrm{en} \% 20$ casa $\% 20 \mathrm{de} \% 20$ ninas $\% 20 \mathrm{y} \% 20$ ninos $\% 20 \mathrm{durant}$ e $\% 201 a \% 20$ pandemia\%20COVID19.pdf? sequence $=1$

Rivera G. M. \& Salto. Q. A. (2017). Screening del desarrollo psicomotor en niños y niñas que asisten a los centros infantiles del buen vivir PATAMARCA II Y HERMANN GMEINER en el periodo del año 2017. Recuperado de https://dspace.ucuenca.edu.ec/bitstream/123456789/28744/1/PROYECTO\%20D E\%20INVESTIGACION.pdf 
Rubio Ponce, R., García Fernández, D. A., \& Cervantes Hernández, N. (2011). Intervención psicomotriz en el área personal/social de un grupo de educación preescolar. Revista complutense de educación. Recuperado de https://redined.educacion.gob.es/xmlui/handle/11162/123445

Ruiz, O. E. B., Sacán, J. E. G., \& Vintimilla, J. M. C. (2021). La educación inicial virtual en contexto de pandemia COVID-19. Aciertos y desafíos: Una Aproximación desde la praxis preprofesional de la carrera de Educación Inicial en la Universidad Nacional de Educación. Mamakuna, 16, 77-87.

Sánchez-Reyes, L. G., Ramón-Santana, A. C., \& Mayorga-Santana, V. E. (2020). Desarrollo Psicomotriz en niños en el contexto del confinamiento por la pandemia del COVID 19. Dominio de las Ciencias, 6(4), 203-219. doi: http://dx.doi.org/10.23857/dc.v6i4.1617

Serrano, P., \& Luque, C. de. (2019). Motricidad fina en niños y niñas: Desarrollo, problemas, estrategias de mejora y evaluación. Narcea Ediciones. 